\title{
Redescrição de três espécies de Artemita Walker (Diptera, Stratiomyidae) do Brasil
}

\author{
Alexandre Ururahy-Rodrigues ${ }^{1,2} \&$ José Roberto Pujol-Luz ${ }^{2}$
}

${ }^{1}$ Programa de Pós-graduação em Biologia Tropical e Recursos Naturais (Entomologia), Instituto Nacional de Pesquisas da Amazônia, INPA, CPEN. Avenida André Araújo, 2936, Bairro Petrópolis. Caixa Postal 478, 69.011-970. ururahy @inpa.gov.br.

${ }^{2}$ Departamento de Zoologia, Instituto de Ciências Biológicas, Universidade de Brasília. Campus Darcy Ribeiro, Asa Norte, Brasília, DF, 70910-900.jrpujol@unb.br

\begin{abstract}
Redescription of three species of Artemita Walker (Diptera, Stratiomyidae) from Brazil. The Stratiomyidae genus Artemita Walker 1854 is represented in the Neotropical Region by 14 species, 6 of which occur in Brazil. The knowledge on the morphology of the species must still be worked. In the present study, male and female of A. brasiliana Lindner, 1964 and females of A. convexa (Walker, 1854) and A. hieroglyphica (Wiedemann, 1830) are redescribed, with the description of their terminalia.
\end{abstract}

KEYWORDS. Neotropical region; Pachygastrinae; terminalia; morphology; taxonomy.

RESUMO. Redescrição de três espécies de Artemita Walker (Diptera, Stratiomyidae) do Brasil. Os Stratiomyidae do gênero Artemita Walker 1854 estão representados na Região Neotropical por 14 espécies, das quais 6 ocorrem no Brasil. O conhecimento sobre a morfologia das espécies ainda precisa ser trabalhado. Neste estudo, macho e fêmea de $A$. brasiliana Lindner, 1964 e as fêmeas de A. convexa (Walker, 1854) e A. hieroglyphica (Wiedemann, 1830) são redescritos, com descrição de suas terminálias.

PALAVRAS-CHAVE. Região Neotropical; Pachygastrinae; morfologia; terminália; taxonomia.

O gênero Artemita Walker é representado na Região Neotropical por 14 espécies: Artemita amenides (Walker, 1849); A. argentea (Osten Sacken, 1886); A. aurata (Macquart, 1846); A. banski James, 1971; A. bellardii (Giglio-Toss, 1891); A. latifrons James, 1971; A. nana (Bellardi, 1862); A. peruviana Kertész, 1914, A. bequaerti (Curran, 1925); A. centor (Curran, 1934); A. brasiliana Lindner, 1964; A. convexa (Walker, 1854); A. hieroglyphica (Wiedmann, 1830) e A. podexargenteus Enderlein, 1914. As 4 últimas ocorrem no Brasil (Woodley, 2001; Ururahy-Rodrigues, 2004). Apesar das importantes contribuições de Kertész (1914) e James (1971) o conhecimento do grupo ainda é rudimentar, principalmente no que diz respeito à morfologia das terminálias. Apesar da importância da terminália como caráter taxonômico, uma única tentativa de estudo mais minuciosa incluindo a terminália do macho foi feita por James (1971) com A. latifrons, mas o autor não apresentou nenhuma comparação com outras espécies do gênero.

Neste estudo, três espécies do gênero Artemita são redescritas (A. brasiliana; A. convexa, A. hieroglyphica), e são descritas as terminálias masculina e feminina de $A$. brasiliana e as terminálias femininas de A. convexa, $A$. hieroglyphica.

\section{MATERIALE MÉTODOS}

Foram examinados 29 exemplares de A. brasiliana, 3 de $A$. hieroglyphica, e 3 de $A$. convexa. Para o estudo da terminália os abdomes foram seccionados transversalmente na altura do primeiro segmento e diafanizados em ácido lático glacial à quente por 30 minutos. As peças foram dissecadas em glicerina e montadas em lâminas permanentes com bálsamo do Canadá ou acondicionadas em tubos plásticos do tipo "microvial". As medidas de comprimento total não incluem as antenas. A terminologia empregada nas redescrições seguiu McAlpine et al. (1981). A terminologia empregada nas redescrições das faixas do mesonoto foi proposta por Enderlein (1914) e a das descrições das estruturas das terminálias por Woodley (1987). Não houve a necessidade do exame do material tipo das espécies estudadas. As redescrições apresentadas tem a intenção de complementar as já existentes, como uma tentativa de fornecer descrições mais completas, incluindo informações sobre a genitália de machos e fêmeas e de maneira comparativa. Informações sobre os tipos foram apresentadas na lista de sinônimos e referências das espécies de Artemita no catálogo de Woodley (2001). Nenhuma das dissecções resultou em total sucesso na obtenção de todas as estruturas da espermateca e em A. brasiliana nenhum dos componentes foi observado, apesar da técnica ser bastante utilizada em outros grupos de Stratiomyidae.

Todo o material examinado está depositado no Museu de Zoologia da Universidade de São Paulo - MZSP.

\section{RESULTADOS}

\section{Artemita brasiliana Lindner, 1964}

Macho: comprimento total: 4,2 - 5,9 mm (Figs. 1-8)

Cabeça com forma geral semi-esférica, em vista dorsal semicircular (Fig. 1). Olhos castanho-avermelhados, com 
poucos pêlos. Fronte (fr) ligeiramente divergente no sentido da face (fc). Antenas com escapo e pedicelo castanho-claros com escassa pilosidade preta; flagelo com oito flagelômeros castanho-escuros com diminutos pêlos pretos, o oitavo clavado com pequenas cerdas apicais pretas. Palpos castanhoescuros com dois segmentos, o primeiro, cerca de duas vezes menor, o segundo com grandes pêlos da mesma cor. Face castanho-clara, gibosa em vista frontal,(Fig. 1).

Tórax em vista dorso-lateral (Fig. 2) com as faixas de pilosidade humerais $(\mathrm{h})$ fusionadas às pós-humerais (ph); faixas pré-alares (pal) fusionadas as dorso-centrais (dc); dorsocentrais largas e fusionadas entre si logo após a sutura transversal, todas prateadas; faixa mediana (m) e dorsais (d) pretas, reduzidas, a primeira terminando antes da sutura transversal com a margem posterior aguda; faixas dorsais com pêlos cor de bronze. Asas hialinas, exceto pelo pterostigma e por manchas castanho-escuras no ápice das células r2 e m; células br e bm inconspícuas; células r-m e cup presentes; célula dm pequena, subtrapezoidal e de localização mediana; halteres alaranjados.

Abdome semicircular em vista dorsal, com cinco segmentos visíveis e cobertos com diminutos pêlos pretos, exceto, por uma mancha formada por pilosidade prateada, dourada ou com ambas, estendendo-se do primeiro ao quinto tergitos. Tegumento preto exceto por duas manchas elípticas, avermelhadas nas margens laterais do primeiro, segundo e terceiro tergitos. Terminália com o hipândrio (hipd) (Figs. 4, 5 e 6) em vista dorsal com apódemas gonocoxais (apod goncx) ligeiramente sinuosos e extremidades arredondadas. Guia edeagal (gui edea) curta e ligeiramente bilobada. Gonóstilos (gonst) subtriangulares com a base larga e ápice delgado, ligeiramente recurvado para o interior e para cima em vista lateral (Fig. 6). Edeago com apódema edeagal menor e mais delgado; apódema ejaculador mais largo em vista dorsal e em vista lateral formando um ângulo fechado em relação ao edeago; bainha parameral (bp)semi-retangular e translúcida com localização mediana (Figs. 7 e 8).

Fêmea: comprimento total: 4,6 - 6,1 mm (Figs. 9-12)

Cabeça com forma geral semi-esférica, em vista dorsal semicircular (Fig. 9). Olhos menos pilosos do que no macho. Occipício proeminente, alcançando a margem posterior do triângulo ocelar em vista lateral. Triângulo ocelar castanhoescuro para preto, ocelos grandes, o anterior maior. Fronte subparalela; placas fronto-orbitais proeminentes castanhoamareladas e cobertas de pilosidade prateada. Face gibosa, castanho-clara margeada por duas faixas distintas, a primeira delgada, glabra, castanho-clara e inconspícua a segunda, mais larga, castanho-escura, com pêlos da mesma cor. Antenas como no macho; gena com coloração e pilosidade semelhantes às placas fronto-orbitais.

Tórax em vista dorso-lateral (Fig. 10) tem o mesonoto com as faixas de pilosidade sempre prateadas, faixas humerais e pós-humerais fusionadas; faixas dorsocentrais livres sem fusionamentos com as faixas pré-alares, o que não ocorre no macho. Asas como no macho; halteres alaranjados.

Terminália com a margem posterior do oitavo tergito em forma de W (Figs. 11, 12); nono tergito (tg 9) longo. Furca com processo anterior (pa) acuminado; abertura anterior cordiforme; processo horizontal presente atravessado pela abertura mediana; processos postero-laterais ( $\mathrm{ppl}$ ) convergentes, membranosos, pouco esclerotinizados (Fig. 12). Espermatecas não foram estudadas.

Material examinado (12 machos e 17 fêmeas, MZSP): Brasil, Santa Catarina, Nova Teutônia, $\left(27^{\circ} 11^{\prime} \mathrm{S} / 52^{\circ} 23 \mathrm{~L}\right.$ ', 300-500 m) (Fritz Plaumman),VIII-1944, 2 machos; X-1944, 1 macho; XII-1964, 1 macho; X-1957, 1 fêmea; X-I. 1967, 1 fêmea; 10-IV-1971, 1 fêmea X1971, 3 machos, Rio Grande do Sul, Fortalesa, VIII-1951 (F. Plaumamm) (Parátipos), 5 machos e 14 fêmeas.

Distribuição geográfica: Brasil (Santa Catarina, Rio Grande do Sul).

\section{Artemita hieroglyphica (Wiedemann, 1830)}

Fêmea: comprimento total em milímetros: 6,3 - 6,8 (Figs. 1317).

Cabeça como em A. brasiliana e A. convexa (Figs. 1, $13 \mathrm{e}$ 18).

Tórax em vista dorso-lateral (Fig. 14) com as faixas de pilosidade do mesonoto dorso-centrais fusionadas às humerais $(\mathrm{dc}+\mathrm{h})$, pós-humerais fusionadas às pré-alares $(\mathrm{ph}+\mathrm{pal})$ todas douradas e estreitas. Faixa mediana e as dorsais com pêlos pretos, inconspícuos em relação à coloração de fundo. Escutelo preto, com quatro espinhos, coberto com pequenos pêlos pretos exceto pela margem posterior, com pêlos dourados e maiores. Pernas com fêmures e tíbias castanho-escuros avermelhados, mas com os ápices mais claros; metatarsos e tarsos castanho-claros, amarelados e com pêlos da mesma cor. Asas como na espécie anterior, exceto pelo pterostigma e por mancha castanho-escura nas células $\mathrm{r} 4, \mathrm{r} 5$ e $\mathrm{m} 1$ com a forma de uma faixa transversal; células br e bm inconspícuas; célula cup presente; célula dm pequena, sub-trapezoidal e de localização mediana; halteres alaranjados.

Abdome como em A. brasiliana. Terminália com a margem posterior do oitavo tergito com forma de "W", e arredondada (Figs. 16 e 17); furca com processo anterior agudo; abertura mediana com forma semitriangular e processos póstero-laterais longos (Fig. 17). Espermatecas: (Fig. 15) com dois ductos capsulares (dca) conectados a duas válvulas (v) também conectadas a dois ductos expulsores (de) incompletos e um ducto comum; os ductos capsulares delgados, corrugados, com a base ligeiramente mais larga, a superfície atravessada por canalículos glandulares irregulares e com glândulas filiformes, mais numerosas no ápice do ducto; válvulas geniculadas; ductos expulsores, estriados horizontalmente e revestidos por espessa camada membranosa translúcida na parte próxima à válvula, e na parte próxima ao ducto comum, membranosos e inconspícuos; ducto comum, largo, membranoso e atravessando a abertura mediana da furca no sentido ventro-dorsal.

Material examinado: (3 fêmeas, MZSP) Brasil, Amapá, Serra do Navio, (Bicelli), X-1957, 1 fêmea. Pará, Belém, Porto da Sesp, IX 

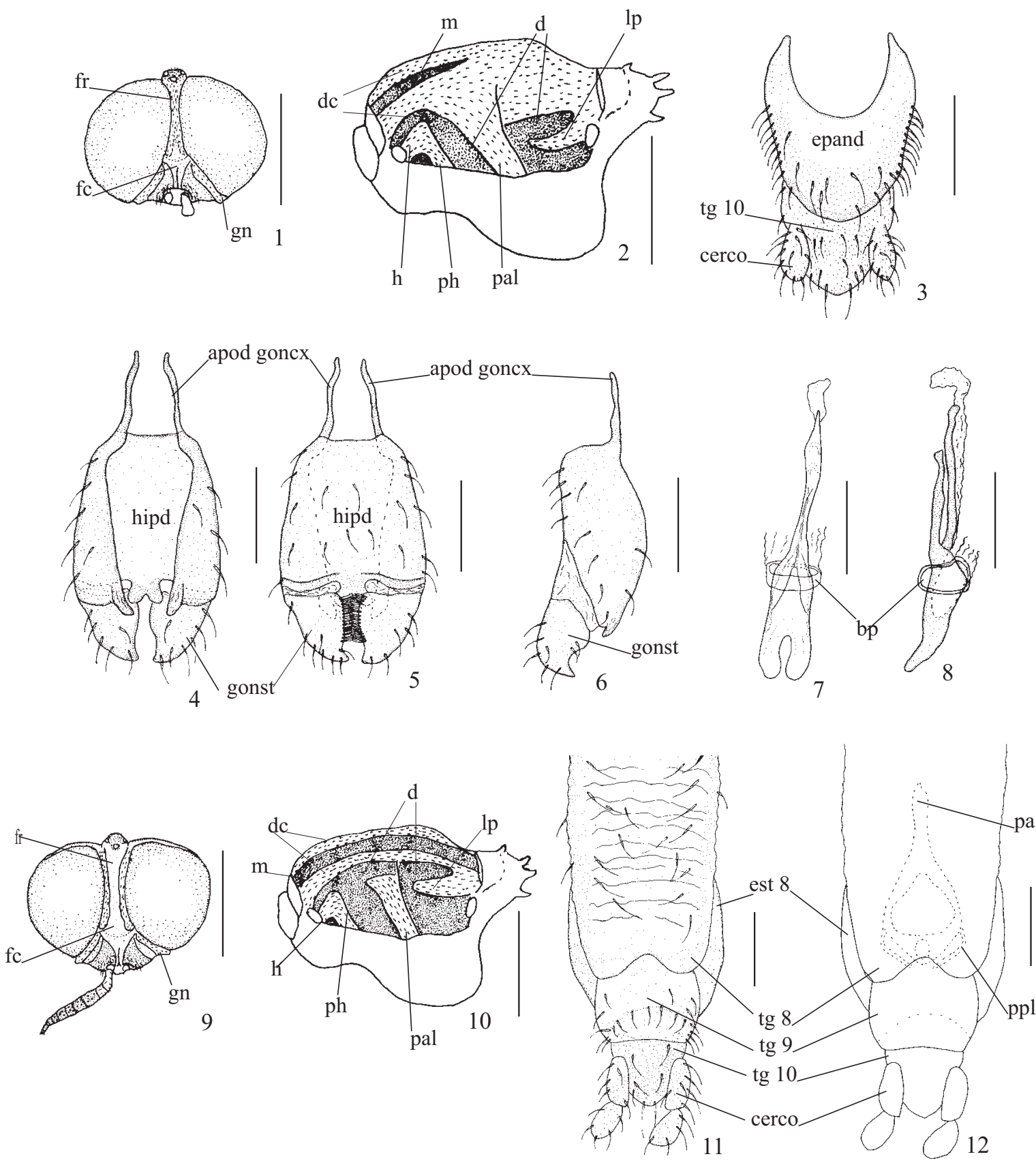

Figs. 1-12. 1-8, Artemita brasiliana Lindner, macho; 1, Cabeça vista frontal; 2, Tórax, vista dorso-lateral; 3-6, Terminália; 3, Epândrio, décimo tergito e cercos vista dorsal; 4-6, Hipândrio+gonocoxitos e gonóstilos; 4, vista dorsal; 5, vista ventral; 6, vista lateral; 7-8, Edeago; 7, vista dorsal; 8, vista lateral; 9-12, Artemita brasiliana Lindner, fêmea; 9, Cabeça, vista frontal; 10, Tórax, vista dorso-lateral; 11, terminália, vista dorsal; 12, terminália e furca em vista dorsal. Legendas: apod goncx = apódema gonocoxal; bp = bainha parameral; $\mathrm{d}=$ faixas do mesonoto dorsais; $\mathrm{dc}=$ faixas do mesonoto dorso-centrais; epand = epândrio; est 8=oitavo esternito; $\mathrm{fc}=$ face; fr $=$ fronte; gn = gena; gonst $=$ gonóstilo; gui edea= guia edeagal; $\mathrm{h}$ = faixas do mesonoto humerais; hipd = hipândrio $\mathrm{lp}=$ faixas do mesonoto longitudinais posteriores; pal $=$ faixas do mesonoto préalares; $\mathrm{ph}=$ faixas do mesonoto pós-humerais; $\operatorname{tg} 8=$ oitavo tergito; $\operatorname{tg} 9=$ nono tergito; tg $10=$ décimo tergito. Barra de escala: Figs $1,2,9$ e 10 $=1 \mathrm{~mm}$; Figs. 3, 4, 5, 6, 11 e $12=0,2 \mathrm{~mm}$; Figs. 7 e $8=0.05 \mathrm{~mm}$.

1969, 1 fêmea. Amazonas, Maturacá, alto rio Canaburi, (J. Bechyni), 23-X-1962, 1 fêmea.

Distribuição geográfica: Bolívia, Brasil (Amapá, Amazonas, Pará), Costa Rica, El Salvador, Guatemala, Honduras, México, Panamá, Paraguai, Peru, Suriname e Trinidad.

\section{Artemita convexa (Walker, 1854)}

Fêmea: comprimento total: 5,1 - 5,5 mm (Figs. 18 - 23).

Cabeça como nas outras espécies estudadas. (Figs. 1, $13 \mathrm{e}$ $18)$.

Tórax em vista dorsal e dorso-lateral (Fig. 19), com as faixas 

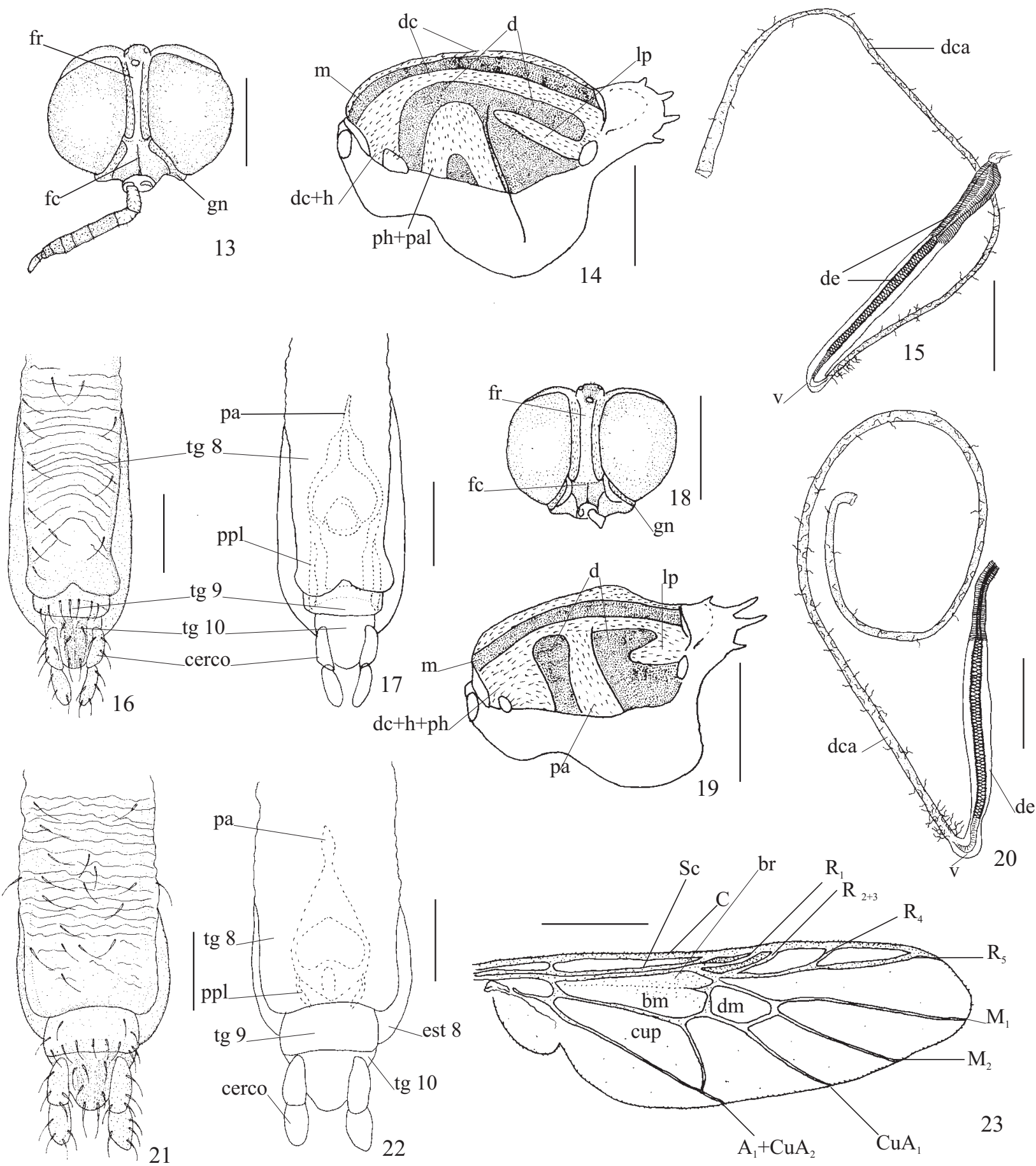

de

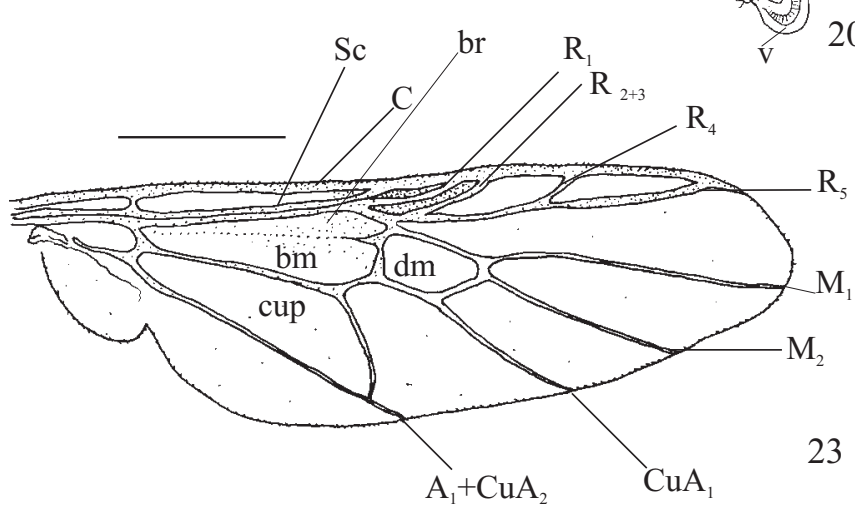

Figs. 13-23. 13-17. Artemita hieroglyphica (Wiedemann), fêmea; 13, Cabeça vista frontal; 14, Tórax, vista dorso-lateral; 15, parte da espermateca; 16, terminália, vista dorsal; 17, terminália e furca em vista dorsal. 18-23. Artemita convexa (Walker); fêmea; 18, Cabeça vista frontal; 19, Tórax, vista dorso-lateral; 20, parte da espermateca; 21 , Terminália, vista dorsal; 22, terminália e furca em vista dorsal. Legendas: $\mathrm{A}_{1}+\mathrm{Cu} \mathrm{A}_{2}=$ primeira veia anal + segunda veia cubital anterior; $\mathrm{bm}=$ célula basal media; $\mathrm{br}=$ célula basal radial; $\mathrm{C}=$ veia costal; $\mathrm{Cu} \mathrm{A}_{1}=$ primeira veia cubital anterior; $\operatorname{cup}=$ célula cubital posterior; $\mathrm{d}=$ faixas do mesonoto dorsais; $\mathrm{dc}=$ faixas do mesonoto dorso-centrais; $\mathrm{dca}=\mathrm{duto}$ capsular; $\mathrm{dc}+\mathrm{h}=\mathrm{faixas}$ do mesonoto dorso-centrais fusionadas às humerais; $\mathrm{dc}+\mathrm{h}+\mathrm{ph}=$ faixas do mesonoto dorso-centrais fusionadas às humerais e às pós-humerais; $\mathrm{dm}=$ célula discal medial; est $8=$ oitavo esternito; $\mathrm{fc}=\mathrm{face}$; $\mathrm{fr}=$ fronte; $\mathrm{gn}=$ gena; $\mathrm{h}=$ faixas do mesonoto humerais; $1 \mathrm{p}=$ faixas do mesonoto longitudinais posteriores; $\mathrm{ph}+\mathrm{pal}=$ faixas do mesonoto pós- humerais fusionadas as pré-alares; $\mathrm{M}=$ Veia Média; $\mathrm{M}_{1}=$ primeira veia média; $\mathrm{M}_{2}=$ segunda veia média; = faixas do mesonoto pós-humerais; $R_{1}=$ primeira veia radial; $R_{2+3}=$ segunda e terceira veias radiais; $R_{4}=$ quarta veia radial; $\mathrm{R}_{5}=$ quinta veia radial; $\operatorname{tg} 8=$ oitavo tergito; tg 9= nono tergito; tg 10= décimo tergito. Barra de escala: Figs. 13, 14, 18, 19 e 23=1 mm; Figs. 15, $16,17,20,21,22=0,2 \mathrm{~mm}$. 
de pilosidade do mesonoto, pós-humerais e humerais fusionadas à base das dorsocentrais $(\mathrm{dc}+\mathrm{h}+\mathrm{ph})$ e as pré-alares fusionadas na região mediana, todas prateadas; faixas dorsais com pêlos pretos e bronze como em A. brasiliana. Asas hialinas e semelhantes às de A. brasiliana, exceto pelo pterostigma e por manchas castanho-escuras no ápice das células r2 e m; células br e bm inconspícuas; células r-m e cup presentes; célula dm pequena, subtrapezoidal e de localização mediana; halteres alaranjados (Fig. 23).

Abdome semelhante a A. brasiliana e A. hieroglyphica. Terminália como em $A$. brasiliana exceto por apresentar a margem posterior do oitavo tergito reta (tg 8) (Figs. 21; 22). Furca com processo anterior acuminado semelhante a $A$. brasiliana (Fig. 22); abertura anterior cordiforme, processo horizontal com a margem posterior dobrada para cima, encobrindo parcialmente a abertura mediana, esta semi-elíptica semelhante à de A. brasiliana (Fig. 22); processos posterolaterais delgados e convergentes e unidos por membrana em condição semelhante às demais espécies (Fig. 22). Espermatecas como em A. hieroglyphica.

Material examinado: (3 fêmeas, MZSP) Brasil, Amapá, Rio Amapari, (J. Lane), 9-X-1957, 1 fêmea. Serra do Navio, 19-X-1957, 1 fêmea; 24-X-1957, 1 fêmea.

Distribuição geográfica: Brasil (Amapá), Costa Rica, Peru.

Agradecimentos. Ao Conselho Nacional de Desenvolvimento Científico e Tecnológico (CNPq) pela bolsa concedida no Programa de Pós-graduação em Biologia Tropical e Recursos Naturais do Instituto
Nacional de Pesquisas da Amazônia (INPA), processo 141747/2004-8 (AUR) e pela bolsa de produtividade em pesquisa, processo 3031195/ 2004-5 (JRPL). À Dra. Eliana Marques Cancello (MZSP) pelo empréstimo do material. Ao Programa de Pós-graduação em Biologia Animal da UFRRJ, onde parte deste trabalho foi desenvolvido. Ao Dr. José Albertino Rafael (INPA) e ao M.Sc. Fabio Siqueira Pitaluga de Godoi (INPA) pela revisão deste manuscrito.

\section{REFERÊNCIAS}

Enderlein, G. 1914. Dipterologische Studien. VIII. Zur Kenntis der Stratiommyiden.Unter Familien mitzästiger Media Pachygasterinae, Lophotelinae und Prosocrhysinae. Zoologischen Anzeiger 43: 289-315.

James, M. T. 1971. The South American species of Artemita. Jounal of the Kansas Entomological Society. 44: 59-70, figs. 1-5.

Kertész, K. 1914. Vorarbeiten zu einer Monographie der Notacanthen. XXIV. Über die Gattung Artemita Walk. Sens. Lat. Annales historico-naturales Musei Nationalis Hungarici 12: 449557.

McAlpine, J. F.; B. V. Peterson; G. E. Shewell; H. J. Teskey; J. R. Vockroth \& D. M. Wood. 1981. Manual of Neartic Diptera. Vol.I. Research Branch, Agriculture Canada I-vi: 1-674.

Ururahy-Rodrigues, A. 2004. Artemita bicolor Kertész, novo sinônimo de Artemita podexargenteus Enderlein, (Diptera, Stratiomyidae) com notas nas terminálias masculina e feminina. Revista Brasileira de Zoologia 21: 397-402.

Woodley, N. E. 1987. The Afrotropical Pachygastrinae genera Ashantina Kertész and Meristomenigina James, with two new generic synonyms (Diptera, Stratiomyidae) Proceedings of the Entomological Society of Washington 89: 103-121.

Woodley, N. E. 2001. A World Catalog of the Stratiomyidae (Insecta, Diptera). Myia 11: 1-475. 